\title{
CHRONIC ARACHNOIDAL CYSTS OF THE MIDDLE CRANIAL FOSSA AND THEIR RELATION TO TRAUMA
}

\author{
BY
}

\section{P. TIBERIN and J. GRUSZKIEWICZ}

From the Department of Neurosurgery of the Rothschild Hadassah Hebrew University Hospital, Jerusalem, Israel

Large accumulations of clear fluid in the cranial cavity, overlying, compressing, displacing, or replacing limited regions of the cerebral hemispheres, are a heterogeneous group of most unusual spaceoccupying lesions.

According to their apparent location in the subdural or subarachnoid spaces and possible aetiology (focal inflammatory processes, congenital anomalies, trauma of meninges), these fluid-filled cavities or cysts, usually walled off by arachnoidal adhesions, have been described by Abbott (1933), Claude (1933), Okonek (1938), Soule and Whitcomb (1946), Taveras and Rouschoff (1953), and Zehnder (1938) under a variety of terms.

Even though considerable confusion still exists in regard to the location and actual pathogenesis of the arachnoidal cysts, there is general agreement as to the origin of the cystic fluid of which the macro- and microscopic appearances and chemical contents have been found to be quite similar to those of the cerebrospinal fluid. Opinions are also agreed on the potential pressure effects, manifested in particular cases of large collections of fluid, by localized thinning and bulging of the skull, and signs of brain compression.

Although cranial enlargement, together with a subdural accumulation of fluids, was mentioned by Davidoff and Dyke as early as 1938, the first to draw attention to the close relationship that exists between large arachnoidal cysts and the associated localized thinning and bulging of the skull was Childe (1953). The latter author described as 'chronic subdural hygroma' eight such cysts and emphasized their frequent localization in the middle fossa.

This particular type of arachnoidal cyst has, as a rule, a prolonged and relatively benign course, is clinically silent, and usually produces few, if any, symptoms in spite of insidious but progressive enlargement.

As these cysts are most prevalent in children, in whom clinical evaluation is often difficult, their diagnosis is frequently obscure for many months or years and some cases may even escape detection altogether.

At times, because of size and clinical behaviour, $\vec{\circ}$ they may produce similar effects to those noted with any slowly expanding lesion and the correct nature $\vec{\sigma}$ of the disease process is, therefore, made either at surgery or incidentally at post-mortem examination.

As we have recently had the opportunity to treat i a young patient harbouring such a huge subarachnoidal cyst, and because of the paucity of the clinical experiences recorded, the report of tho clinical and operative features of this case is pre sented as material which may shed some light on tire controversial problem of the origin and pathogenesis 8 of this condition.

\section{Clinical History}

Case GA (No. 128882). -A boy aged 10 was admitted on August 21, 1959, with a long history of intermittent bitemporal headaches which had recently increased in intensity.

The family history contributed nothing.

The boy's birth and subsequent development were relatively normal, and, except for three successive attacks of pneumonia at the age of 2 , he had had no previous $\overrightarrow{\vec{B}}$ illnesses.

At the age of 3 he had fallen out of his bed and lost consciousness for a short time. No exact details on this point could be obtained, but apparently there were no after-effects until three months later, when he was first noticed to have a bony prominence anterior to and above the right ear. From then on the child became emotionally unstable and gradually developed enuresis, showed 3 outbursts of aggressive and hyperkinetic behaviour, and $\bar{\delta}$ was generally rather difficult to manage. He had no convulsions, attacks of unconsciousness, or automatisms.

At the age of 5 , left convergent strabismus became $D$ evident and from then on the child started to wear glasses; nevertheless, the visual acuity in the left eye progressively deteriorated.

He reached school at the age of 7 , but failed to make $N$ proper progress, and at that time began to complain of $\mathrm{N}$ 


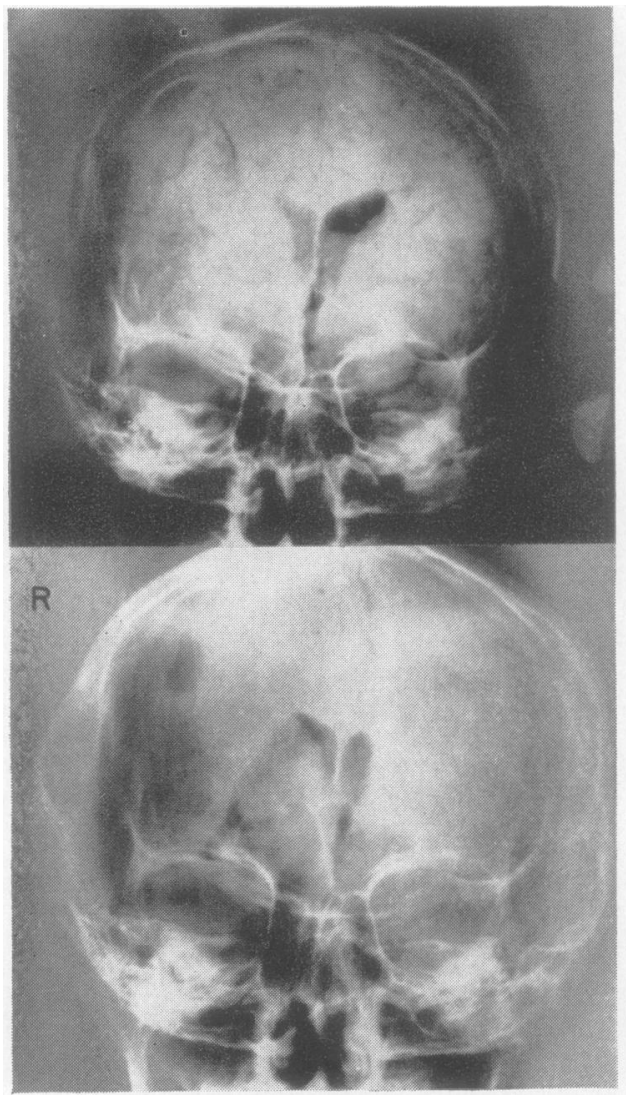

(a)

(b)

FIG. 1.-(a) Antero-posterior pneumoencephalogram showing slight shift of the septum pellucidum to the left and asymmetry of lateral ventricles, the right being smaller than the left.

(b) Right temporal pneumoencephalogram (short view) showing elevation of the temporal horn tip and increased distance between it and the inner aspect of the enlarged skull, but normal relation of the former to the midline structures.

headache localized in both temporal regions. As they had lately become more frequent and had gradually increased in intensity, further attention was paid to the bulging of the right temple and it was thought at that time that it had become more marked.

Physical examination revealed a pale-looking boy of lower level-normal intelligence. The right anterior temporal region was markedly prominent. The chest was clear to percussion and auscultation. The heart was not enlarged, its beats were regular and no murmurs were audible. Blood pressure was $115 / 70 \mathrm{~mm}$. Hg. The abdomen was soft, the liver and spleen were not palpable, and the genitalia were normal.

The neurological examination was negative except for hyperactive deep reflexes on the left side.

Eye examination revealed signs of hypermetropic astigmatism. Visual acuity with the patient wearing glasses was 20/20 in the right eye $(+6.0)$ and 20/200 in the left $\left(+6 \cdot 5=2 \cdot 0 \mathrm{cyl} .80^{\circ}\right)$.
Laboratory findings of blood and urine were within normal limits and a radiograph of the chest was normal.

Radiographs of the skull showed pronounced outward bulging of the right temporal region with thinning of the bone, elevation of the right lesser wing of the sphenoid, and marked forward enlargement of the right middle cranial fossa. There were no radiological signs of generalized increased intracranial pressure. An E.E.G. showed decreased amplitude, slow wave focus over the right temporal region. Pneumoencephalography revealed that the septum pellucidum was slightly shifted to the left, the right lateral ventricle being smaller than the left. Moderate elevation of the right temporal horn

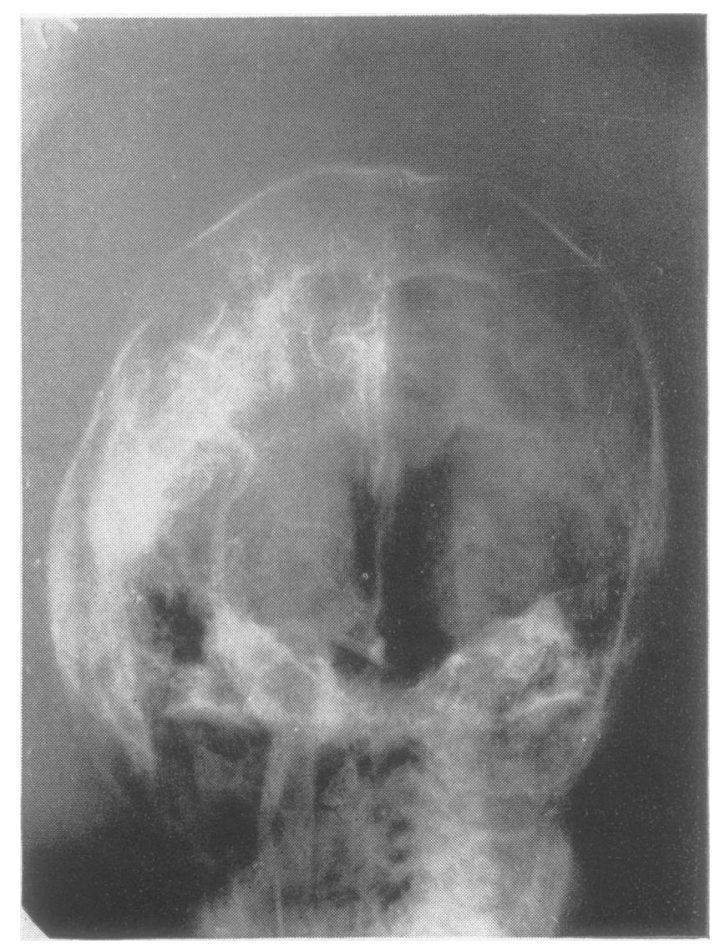

FIG. 2.-Antero-posterior right carotid angiogram showing slight shift of the anterior cerebral artery to the left and marked displacement of the middle cerebral artery upward and medialward. Note the obvious asymmetry of the skull.

tip was apparent, the length of the temporal horns being obviously unequal. Moreover, the subarachnoid pattern over the right temporal lobe was lacking and the distance between the inner table of the bulged right temporal squama and the right temporal horn was markedly greater than on the left side (Fig. 1). The cerebrospinal fluid removed during this procedure was clear and contained $27 \mathrm{mg}$. of protein per $100 \mathrm{ml}$. Right carotid angiography demonstrated that the anterior cerebral artery was slightly displaced to the left and that the middle cerebral artery and its branches were markedly displaced medially and upward. Between the inner table 
FIg. 3.-(a) Operative field after aspiration of the cystic fluid to show the antero-lateral aspect of the temporal lobe in relation to the enlarged middle fossa. (b) The tip of the bayonet forceps is pointing to the delicate trabecular
which potential communication existed between the cyst's cavity and the general subarachnoid space.

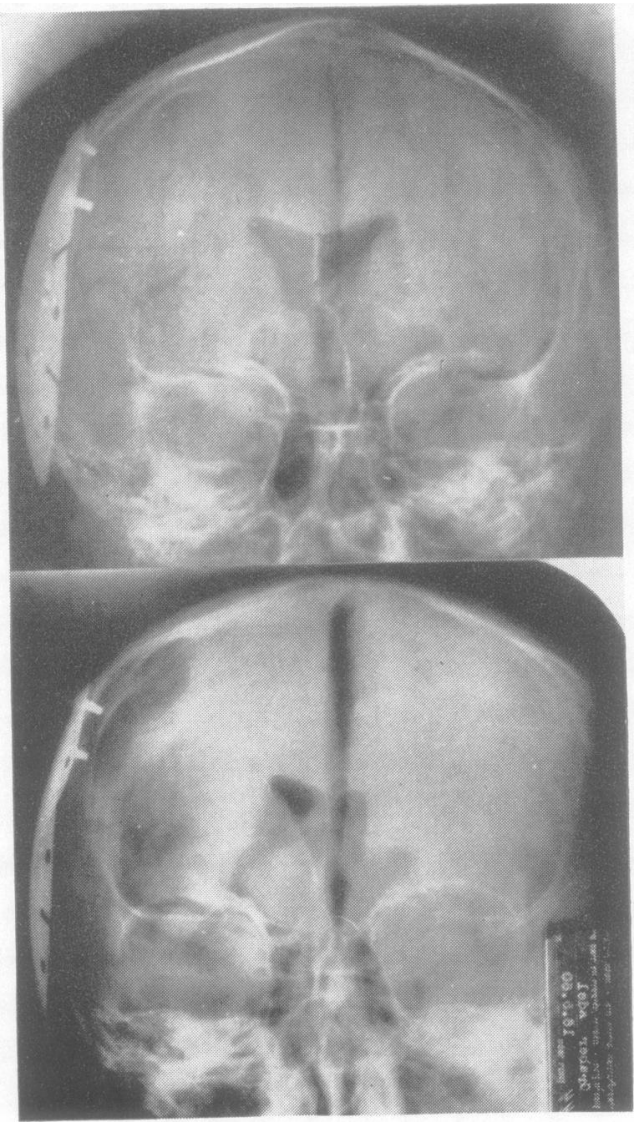

(a)

(b)

FIG. 4.- (a) Post-operative pneumoencephalogram showing slight symmetrical dilatation of the lateral ventricles and their shift to the side of the lesion.

(b) Right temporal shot view to show normal length of the temporal horn. 
of the skull and the brain there was an avascular area (Fig. 2). In the lateral view, the elevation of the middle cerebral vessels was not as pronounced as the anteroposterior film suggested.

The clinical findings raised the possibility of a longstanding, slowly progressive intracranial expanding lesion and a pre-operative diagnosis of chronic posttraumatic arachnoidal cyst was taken into consideration.

Operation.-Through a linear incision, a trephine opening was made over the middle of the bulged temporal bone, which was found to be of eggshell thickness.

After enlarging the burr opening, a circular skull defect $6 \mathrm{~cm}$. in diameter was obtained exposing a bluish, tense dura mater. It was punctured and about $40 \mathrm{ml}$. of clear colourless fluid was withdrawn. The dura was then incised and opened in the usual manner, resulting in the exposure of a huge thin-walled cystic cavity that occupied the entire anterior portion of the middle cranial fossa as well as the outer third of its middle part. It contained as much as $150 \mathrm{ml}$. of clear cerebrospinal-like fluid of which the protein content was $53 \mathrm{mg}$. per $100 \mathrm{ml}$.

After complete evacuation of the fluid, the size of the right temporal lobe appeared to be rather small in relation to the markedly enlarged middle fossa, but its configuration, including the gyral pattern, was of normal aspect. There was no lining covering the temporal lobe and it was evident that the membrane representing the lateral wall of the cyst was continuous with the arachnoid overlying the cerebral cortex.

A delicate trabecular connective tissue network connected this membrane to the pia of the temporal lobe $4 \frac{1}{2} \mathrm{~cm}$. posteriorly from its tip, and along the posterior part of the Sylvian fissure, allowing some communication between the cyst's cavity and the subarachnoid space overlying the neighbouring cerebral convexity (Fig. 3). The empty anterior portion of the middle fossa measured $4.5 \mathrm{~cm}$. in length and $3.5 \mathrm{~cm}$. in width. The distance between the dura and the convexity of the temporal lobe gradually decreased from its tip backward. The lateral wall of the cyst was partially adherent to the inner lining of the dura from which it could be easily separated, and part of it removed for examination. In order to relieve the tension exerted on the underlying cortex, free communication between the relatively closed cavity of the cyst and the general subarachnoid space was obtained by careful separation of the adhesions between the pia mater and the lateral wall of the cyst. No expansion of the temporal lobe could be observed. After water-tight closure of the dura, the bone defect was covered by a plate of stainless steel and the wound closed in the usual fashion.

The histological examination showed a membrane similar to arachnoidea, with scattered psamona bodies.

The child's post-operative course was uneventful, the headaches did not recur, the enuresis completely disappeared for a period of three months, when it recurred, appearing at longer intervals.

Pneumoencephalography performed nine months after operation showed symmetrical dilatation of the lateral ventricles and equal length of both temporal horns (Fig. 4).
The clinical improvement was accompanied by the disappearance of the abnormal pattern in the E.E.G.

\section{Discussion}

Large, pseudo-tumoral, chronic arachnoidal cysts of the middle cranial fossa accompanied by local enlargement of the skull represent a clinico-pathological entity which, surprisingly, has seldom been reported in the medical literature.

Besides the cases presented by Childe at the 52nd annual meeting of the American RoentgenRay Society and published in 1953, only a few cases of this particular type of lesion had been previously reported.

In 1955, two additional cases of localized collections of fluid, accompanied by thinning and bulging of the temporal squama as well as enlargement of the middle cranial fossa, were reported by Robinson, who emphasized the apparent developmental origin of these cysts and their unequivocal location in the subarachnoid space. The ratity of this condition is stressed by the fact that in his review of the world literature, he was unable to find more than 15 similar cases up to that time. His series does not include the case of Briusova (1951) reported in the Russian medical literature.

More recently, the developmental origin of some of the large arachnoidal cysts has been supported by the pathological findings, incidentally encountered in three cases at necropsy by Starkman, Brown, and Linell (1958).

In the same year, another five cases were reported by Robinson (1958), in three of which large collections of fluid resembling cerebrospinal fluid localized in the middle cranial fossa were associated with bulging of the overlying temporal bone. He suggested for this condition the term 'external hydrocephalus', which, in his opinion, was secondary to the agenesis of the temporal lobe.

That such a conclusion may not be warranted in all cases is suggested by the fact that signs of previous subdural bleeding were found in Childe's (1953) Cases 2 and 3 as well as in those of Cohen (1927), Hardman (1939), and Pertuiset and Berdet (1952), included by Robinson (1955) in his first series of cases. Moreover no local enlargement of the skull was present in Case 4 of Robinson (1958) as well as in the cases of Starkman et al. (1958), while in Robinson's (1958) Case 7 no neuroradiological investigations were performed at all, nor was the condition confirmed by surgery. The above-mentioned cases did not fulfil thereby either the radiological or pathological criteria characteristic of the type of arachnoidal cyst under consideration. 
Analysis of the remaining 17 recorded cases showed that bulging of the temporal squama was the main complaint in all but one instance. In five of the cases this was the only change revealed in radiographs of the skull, while in the others, including our own case and the one in which no bulging of the temporal bone was mentioned, enlargement of the middle cranial fossa was present as well.

In only seven of the above cases did symptoms and signs of variable neurological deficit dominate the clinical picture. In reviewing all the recorded supratentorial arachnoidal cysts, we came to the conclusion that two factors at least are responsible for their occurrence, namely, a primary cerebral developmental defect, in which limited regions of the brain are missing and simply replaced by cerebrospinal fluid, the resulting fluid-filled cavities being in free communication with the general subarachnoid space. In these cases, the pressure of the collected fluid is not increased and consequently no secondary cranial changes occur. Moreover, associated porencephaly, hydrocephalus, or other malformations of the brain are usually present. Secondly, a mild closed head injury during early childhood, in which cases the clinical picture is characterized later in life by symptoms of cerebral compression and signs of localized bone changes, as in our patient.

Although there is no objective evidence to support the proposition that trauma of the head in early life is the responsible factor for this condition, such a possibility cannot be denied. It is generally thought that during a head injury the supratentorial structures of the brain are able to move through the tentorial openings. The temporal lobes, however, are withheld by the bony floor of the middle cranial fossa as well as by the tentorium itself. It is presumed that during the shifting of the brain in the direction of the impact, the contusion of the temporal lobe tip or of the hippocampal gyrus is accompanied by a small subarachnoid haemorrhage that later on becomes partially encapsulated in the anterior part of the middle fossa. As the protein molecules disintegrate, there is an increase in the osmotic pressure, and blood serum as well as cerebrospinal fluid is drawn in. In the course of time, as communication between this collection and the general subarachnoid space is partially maintained, absorption of the blood is possible and further accumulation of cerebrospinal fluid permitted.

It appears probable that the thinning and bulging of the temporal bone as well as the forward enlargement of the middle fossa occurs as a result of the continuous intracranial arterial pulsating pressure, transmitted to the endocranium by the local accumulation of fluid. This view is consistent with the ${ }_{\mathbb{\infty}}^{Z}$ opinion of List and Pendergrass expressed in the discussion following the paper of Childe (1953). Ito is assumed that these changes occur during earlyo childhood, when the middle fossa may adapt itselfo to the enlarging cyst, similar to the moulding of the inner table of the skull by the growing brain.

When a history of trauma cannot be ascertained the clinical diagnosis of chronic arachnoidal cyst is virtually impossible. Since superficially situated gliomas (Jupe, 1938; Orley, 1949), or subduraf haematomas (Davidoff and Dyke, 1938), or chronic subdural hygromas (Hardman, 1939) may at times produce in children the same bony changes as are found in cases of arachnoidal cysts, one can only assume that a tumour or other intracranial slowlyes expanding lesion is present. In such cases even $\vec{b}$ pneumoencephalography or angiography may beuseless and only the placing of an exploratory burrw hole over the bulging of the skull will reveal the reas nature of the lesion.

There are as yet differences in opinion concerning the necessity to open and drain these cysts. There is no doubt that when the condition is asymptip matic, as it usually is in cases in which the che results from a developmental defect of the brain $\$$ meninges, there are no indications at all for surgess However, in cases like our own, in which the cसsto plays the role of an expanding lesion, complete aspiration of the fluid and careful separation of the arachno-cortical adhesions is, in our opiniogn. mandatory in order to improve the actual clinifges syndrome and prevent further cerebral damage from reaccumulation of fluid.

The mild post-operative symmetrical dilatation of the lateral ventricles and their slight shift to the side of the previous lesion, as revealed in our patien by pneumoencephalography, is in keeping with the concept of an 'ex-vacuo' expansion of the brain. $\stackrel{\vec{F}}{\Rightarrow}$

Moreover, the equality in length of both tempora? horns is an undoubted indication that, in thispatient, there was no deficiency whatsoever of the temporal lobe. In our opinion this objective finding is a strong argument against the generally accepted view of a developmental origin for the chronie arachnoidal cysts of the middle fossa associated with the aforementioned bone changes.

\section{Summary and Conclusions}

A case of chronic pseudo-tumoural large sub을 arachnoid cyst of the middle cranial fossa in 8 10-year-old boy has been presented in order to: illustrate this most unusual benign intracraniaf space-occupying lesion, in which trauma of the head seemed to be the aetiological factor. 
While the actual mechanism is still debatable, subarachnoid bleeding (in the middle fossa), brain oedema, and any interference with the normal blood-brain barrier's function that might have resulted from the concussive force are to be considered significant contributory factors in the pathogenesis of this cyst.

In a review of the literature, only 17 similar cases have been found. With our own case the total number of the reported cases rises to 18 . Whether these figures represent the true incidence of the condition or not, is doubtful, since chronic arachnoidal cysts are as a rule silent.

The probability that trauma of the head in early life is the responsible aetiological factor of this condition cannot be excluded, in spite of the fact that no history of head injury could be obtained in about $50 \%$ of the above cases. It is assumed that in those cases in which no history of trauma has been mentioned, minor traumas in the past could have been overlooked.

Symptoms varying from headache and irritability to a full temporal lobe syndrome, as present in this case, and local bone changes characterized by thinning and bulging of the temporal squama, as well as widening, deepening, and lengthening of the middle cranial fossa, may develop.

As a rule, the presence of the lesion cannot be established by clinical observation only. Although not pathognomonic for this condition radiographs of the skull, air studies of the ventricular system as well as cerebral angiography, are sufficiently characteristic to help in diagnosis. Ultimately, however, the condition can be diagnosed with certainty only by direct inspection.

The treatment of this lesion, in spite of its benign evolution, is surgical and the prognosis is usually good.

An alertness to the possibility of this intracranial, space-occupying lesion may result in an increased number of such cases being disclosed.

Grateful acknowledgement is made to Professor A. J. Beller, Head of the Department of Neurosurgery, for his advice and helpful criticism. Thanks are also due to Mr. G. Mageni and Mr. M. Ivri for the photographs.

\section{REFERENCES}

Abbott, W. D. (1933). J. Iowa St. med. Soc., 23, 315

Briusova, S. S. (1951). Angiographia Mazga, Izdatielstwo Akademi Medicinskich NaUk, U.S.S.R., p. 153.

Childe, A. E. (1953). Amer. J. Roentgenol., 70, 1.

Claude, H. (1933). Rev. neurol., 1, 824.

Cohen, I. (1927). Arch. Neurol.'Psychiat. (Chicago), 18, 709.

Davidoff, L. M., and Dyke, C. G. (1938). Bull. neurol. Inst. N.Y., 7,95

Hardman, J. (1939). Brit. J. Radiol., 12, 455.

Jupe, M. H. (1938). Ibid., 11, 146.

Okonek, G. (1938). Zbl. Neurochir., 3, 112.

Orley, A. (1949). Neuroradiology. Thomas, Springfield, Illinois.

Pertuiset, B., and Berdet, H. (1952). Rev. neurol., 87, 538.

Robinson, R. G. (1955). J. Neurosurg., 12, 345.

(1958). Brit. J. Radiol., 31, 691.

Soule, A. B., and Whitcomb, B. B. (1946). Arch. Neurol. Psychiat., 55, 382 .

Starkman, S. P., Brown, T. C., and Linell, E. (1958). J. Neuropath. exper. neurol., 17, 484.

Taveras, J. M., and Rouschoff, J. (1953). J. Neurosurg., 10, 233.

Zehnder, M. (1938). Zbl. Neurochir., 3, 111. 